\title{
Correction to: Capacity analysis of maximal ratio combining over Beaulieu-Xie fading
}

\section{Veenu Kansal $^{1} \cdot$ Simranjit Singh ${ }^{1}$}

Published online: 13 May 2020

(C) Institut Mines-Télécom and Springer Nature Switzerland AG 2020

\section{Correction to: Annals of Telecommunications.} https://doi.org/10.1007/s12243-020-00762-7

Equations 21, 22, and 23 were incorrectly captured in the original manuscript. The correct equations are presented below.
Case 1: Rayleigh fading $(\mathrm{m}=1, \mathrm{~K}=0)$.

The expression in (20) can be reduced to Rayleigh fading by substituting the value of fading parameters, $\mathrm{K}=0$ and $\mathrm{m}=1$ as follows:

$C_{\text {ORA }}=B \times \log _{2}(e) \times\left(\frac{1}{\bar{\gamma}}\right)^{L} \times \exp \left(\frac{1}{\bar{\gamma}}\right) \times \sum_{t=0}^{\infty} g_{t} \times \sum_{h=1}^{t+L} \frac{\Gamma\left(-t-L+h, \frac{1}{\bar{\gamma}}\right)}{\left(\frac{1}{\bar{\gamma}}\right)^{h}}$

Case 2: Rician fading $(\mathrm{m}=1, \mathrm{~K}>0)$.

$C_{\text {ORA }}=B \times \log _{2}(e) \times\left(\frac{K+1}{\bar{\gamma}}\right)^{L} \times \exp \left(-K L+\frac{K+1}{\bar{\gamma}}\right) \times \sum_{t=0}^{\infty} g_{t} \times \sum_{h=1}^{t+L} \frac{\Gamma\left(-t-L+h, \frac{K+1}{\bar{\gamma}}\right)}{\left(\frac{K+1}{\bar{\gamma}}\right)^{h}}$
The expression in (20) can be reduced to Rician fading by substituting the value of fading parameters, $\mathrm{K}>0$ and $\mathrm{m}=$ 1as follows:
Case 2: Rician fading $(\mathrm{m}=1, \mathrm{~K}>0)$.

The online version of the original article can be found at https://doi.org/ $10.1007 / \mathrm{s} 12243-020-00762-7$

Simranjit Singh

simranjit@live.com

Veenu Kansal

veenukansal@outlook.com

1 Department of Electronics and Communication Engineering, Punjabi University, Patiala, India 
Case 3: Nakagami-m fading $(\mathrm{m}>0.5, \mathrm{~K}=0)$.

The expression in (20) can be reduced to Nakagami-m fading by substituting the value of fading parameters, $\mathrm{K}=0$ and $\mathrm{m}>$ 0.5 as follows:

$C_{O R A}=B \times \log _{2}(e) \times\left(\frac{m}{\bar{\gamma}}\right)^{L} \times \exp \left(\frac{m}{\bar{\gamma}}\right) \times \sum_{t=0}^{\infty} g_{t} \times \sum_{h=1}^{t+L} \frac{\Gamma\left(-t-L+h, \frac{m}{\bar{\gamma}}\right)}{\left(\frac{m}{\bar{\gamma}}\right)^{h}}$
Similarly, the expressions derived for other adaptive transmission techniques can be deduced as special case of BX fading channel.

The Publisher regrets this error.

The original article has been corrected. 